\title{
Volume-preserving diffeomorphisms with inverse shadowing
}

Manseob Lee*

\section{"Correspondence:}

Imsds@mokwon.ac.kr

Department of Mathematics,

Mokwon University, Daejeon,

302-729, Korea

\section{第 Springer}

\begin{abstract}
Let $f$ be a volume-preserving diffeomorphism of a closed $C^{\infty} n$-dimensional Riemannian manifold $M$. In this paper, we prove the equivalence between the following conditions:

(a) $f$ belongs to the $C^{1}$-interior of the set of volume-preserving diffeomorphisms which satisfy the inverse shadowing property with respect to the continuous methods,

(b) $f$ belongs to the $C^{1}$-interior of the set of volume-preserving diffeomorphisms which satisfy the weak inverse shadowing property with respect to the continuous methods,

(c) $f$ belongs to the $C^{1}$-interior of the set of volume-preserving diffeomorphisms which satisfy the orbital inverse shadowing property with respect to the continuous methods,

(d) $f$ is Anosov.

MSC: Primary 37C50; secondary 34D10
\end{abstract}

Keywords: shadowing; inverse shadowing; weak inverse shadowing; orbital inverse shadowing; Anosov; volume-preserving

\section{Introduction}

Let $M$ be a closed $C^{\infty} n$-dimensional Riemannian manifold, and let $\operatorname{Diff}(M)$ be the space of diffeomorphisms of $M$ endowed with the $C^{1}$-topology. Denote by $d$ the distance on $M$ induced from a Riemannian metric $\|\cdot\|$ on the tangent bundle TM. Let $f: M \rightarrow M$ be a diffeomorphism, and let $\Lambda \subset M$ be a closed $f$-invariant set.

For $\delta>0$, a sequence of points $\left\{x_{i}\right\}_{i=a}^{b}(-\infty \leq a<b \leq \infty)$ in $M$ is called a $\delta$-pseudo orbit of $f$ if $d\left(f\left(x_{i}\right), x_{i+1}\right)<\delta$ for all $a \leq i \leq b-1$. We say that $f$ has the shadowing property on $\Lambda$ if for any $\epsilon>0$, there is $\delta>0$ such that for any $\delta$-pseudo orbit $\left\{x_{i}\right\}_{i \in \mathbb{Z}} \subset \Lambda$ of $f$, there is $y \in M$ such that $d\left(f^{i}(y), x_{i}\right)<\epsilon$ for $i \in \mathbb{Z}$. Note that in this definition, the shadowing point $y \in M$ is not necessarily contained in $\Lambda$. We say that $f$ belongs to the $C^{1}$-interior shadowing property if there is a $C^{1}$-neighborhood $\mathcal{U}(f)$ of $f$ such that for any $g \in \mathcal{U}(f), g$ has the shadowing property.

The shadowing property usually plays an important role in the investigation of stability theory and ergodic theory [1].

Now, we introduce the notion of the inverse shadowing property which is a 'dual' notion of the shadowing property. The inverse shadowing property was introduced by Corless and Pilyugin in [2], and the qualitative theory of dynamical systems with the property was

(C) 2012 Lee; licensee Springer. This is an Open Access article distributed under the terms of the Creative Commons Attribution License (http://creativecommons.org/licenses/by/2.0), which permits unrestricted use, distribution, and reproduction in any medium, provided the original work is properly cited. 
developed by various authors (see [2-7]). In this paper, we introduce the various inverse shadowing properties.

Let $M^{\mathbb{Z}}$ be the space of all two-sided sequences $\xi=\left\{x_{n}: n \in \mathbb{Z}\right\}$ with elements $x_{n} \in M$, endowed with the product topology. For any $\delta>0$, let $\Phi_{f}(\delta)$ denote the set of all $\delta$-pseudo orbits of $f$. A mapping $\varphi: M \rightarrow \Phi_{f}(\delta) \subset M^{\mathbb{Z}}$ is said to be a $\delta$-method for $f$ if $\varphi(x)_{0}=x$, and each $\varphi(x)$ is a $\delta$-pseudo orbit of $f$ through $x$, where $\varphi(x)_{0}$ denotes the 0th component

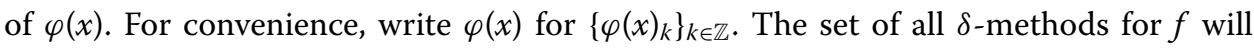
be denoted by $\mathcal{T}_{0}(f, \delta)$. We say that $\varphi$ is a continuous $\delta$-method for $f$ if $\varphi$ is continuous. The set of all continuous $\delta$-methods for $f$ will be denoted by $\mathcal{T}_{c}(f, \delta)$. If $g: M \rightarrow M$ is a homeomorphism with $d_{0}(f, g)<\delta$, then $g$ induces a continuous $\delta$-method $\varphi_{g}$ for $f$ by defining

$$
\varphi_{g}(x)=\left\{g^{n}(x): n \in \mathbb{Z}\right\}
$$

Let $\mathcal{T}_{h}(f, \delta)$ denote the set of all continuous $\delta$-methods $\varphi_{g}$ for $f$ which are induced by a homeomorphism $g: M \rightarrow M$ with $d_{0}(f, g)<\delta$, where $d_{0}$ is the usual $C^{0}$-metric. Let $\mathcal{T}_{d}(f, \delta)$ denote the set of all continuous $\delta$-methods $\varphi_{g}$ for $f$ which are induced by $g \in \operatorname{Diff}(M)$ with $d_{1}(f, g)<\delta$. Then, clearly, we know that

$$
\mathcal{T}_{d}(f) \subset \mathcal{T}_{h}(f) \subset \mathcal{T}_{c}(f) \subset \mathcal{T}_{0}(f),
$$

$\mathcal{T}_{\alpha}(f)=\bigcup_{\delta>0} \mathcal{T}_{\alpha}(f, \delta), \alpha=0, c, h, d$. We say that $f$ has the inverse shadowing property with respect to the class $\mathcal{T}_{\alpha}(f), \alpha=0, c, h, d$, if for any $\epsilon>0$, there exists $\delta>0$ such that for any $\delta$-method $\varphi \in \mathcal{T}_{\alpha}(f, \delta)$ and any point $x \in M$, there is a point $y \in M$, such that

$$
d\left(f^{k}(x), \varphi(y)_{k}\right)<\epsilon, \quad k \in \mathbb{Z}
$$

We say that $f$ has the weak inverse shadowing property with respect to the class $\mathcal{T}_{\alpha}(f)$, $\alpha=0, c, h, d$, if for any $\epsilon>0$, there exists $\delta>0$ such that for any $\delta$-method $\varphi \in \mathcal{T}_{\alpha}(f, \delta)$ and any point $x \in M$, there is a point $y \in M$ such that

$$
\varphi(y) \subset B_{\epsilon}\left(\mathcal{O}_{f}(x)\right)
$$

where $B_{\epsilon}(A)=\{x \in M: d(x, A) \leq \epsilon\}$. Note that if $f \in \operatorname{Diff}(M)$ has the inverse shadowing property with respect to the class $\mathcal{T}_{\alpha}(f)(\alpha=0, c, h, d)$, then by the definition, it clearly has the weak inverse shadowing property with respect to the class $\mathcal{T}_{\alpha}(f)(\alpha=0, c, h, d)$. We say that $f$ has the orbital inverse shadowing property with respect to the class $\mathcal{T}_{\alpha}(f)$, $\alpha=0, c, h, d$, if for any $\epsilon>0$, there is a $\delta>0$ such that for any $\delta$-method $\varphi \in \mathcal{T}_{\alpha}(f, \delta)$ and any point $x \in M$, there is a point $y \in M$ such that

$$
\mathcal{O}_{f}(x) \subset B_{\epsilon}(\varphi(y)) \text { and } \varphi(y) \subset B_{\epsilon}\left(\mathcal{O}_{f}(x)\right) .
$$

Note that if $f$ has the inverse shadowing property with respect to the class $\mathcal{T}_{\alpha}(f)$ ( $\alpha=$ $0, c, h, d)$, then it has the orbital inverse shadowing property with respect to the class $\mathcal{T}_{\alpha}(f)$ $(\alpha=0, c, h, d)$. But the converse does not hold. Indeed, an irrational rotation on the unit circle has the orbital inverse shadowing property but does not have the inverse shadowing 
property with respect to the class $\mathcal{T}_{d}(f)$. We say that $f$ belongs to the $C^{1}$-interior inverse (weak inverse or orbital inverse) shadowing property with respect to the class $\mathcal{T}_{\alpha}(f), \alpha=$ $0, c, h, d$ if there is a $C^{1}$-neighborhood $\mathcal{U}(f)$ of $f$ such that for any $g \in \mathcal{U}(f), g$ has the inverse (weak inverse or orbital inverse) shadowing property with respect to the class $\mathcal{T}_{\alpha}(f), \alpha=$ $0, c, h, d$.

Lee [6], showed that a diffeomorphism belongs to the $C^{1}$-interior inverse shadowing property with respect to the class $\mathcal{T}_{d}(f)$ if and only if it is structurally stable. And Pilyugin [7] proved that a diffeomorphism belongs to the $C^{1}$-interior inverse shadowing property with respect to the class $\mathcal{T}_{c}(f)$ if and only if it is structurally stable. Thus, we can restate the above facts as follows.

Theorem 1.1 Let $f \in \operatorname{Diff}(M)$. A diffeomorphism $f$ belongs to the $C^{1}$-interior inverse shadowing property with respect to the class $\mathcal{T}_{d}(f)\left(\right.$ resp. $\left.\mathcal{T}_{c}(f)\right)$ if and only if it is structurally stable.

In [3] Choi, Lee and Zhang showed that a diffeomorphism belongs to the $C^{1}$-interior weak inverse shadowing property with respect to the class $\mathcal{T}_{d}(f)$ if and only if it satisfies both Axiom A and the no-cycle condition. Moreover, they proved that a diffeomorphism belongs to the $C^{1}$-interior orbital inverse shadowing property with respect to the class $\mathcal{T}_{d}(f)$ if and only if it satisfies both Axiom A and the strong transversal condition. From the above facts, we get the following.

Theorem 1.2 Let $f \in \operatorname{Diff}(M)$.If a diffeomorphism $f$ belongs to the $C^{1}$-interior weak inverse shadowing property with respect to the class $\mathcal{T}_{d}(f)$, then $f$ satisfies both Axiom $\mathrm{A}$ and the nocycle condition. Moreover, iff belongs to the $C^{1}$-interior orbital inverse shadowing property with respect to the class $\mathcal{T}_{d}(f)$, then it is structurally stable.

By the theorem, even though a diffeomorphism is contained in the $C^{1}$-interior of the set of diffeomorphisms possessing the weak inverse shadowing property with respect to the class $\mathcal{T}_{d}(f)$, it does not necessarily satisfy the strong transversality condition.

A periodic point $p$ of $f$ is hyperbolic if $D f^{\pi(p)}$ has eigenvalues with absolute values different from the one, where $\pi(p)$ is the period of $p$. Denote by $\mathcal{F}(M)$ the set of $f \in \operatorname{Diff}(M)$ such that there is a $C^{1}$-neighborhood $\mathcal{U}(f)$ of $f$ such that for any $g \in \mathcal{U}(f)$, every $p \in P(g)$ is hyperbolic, where $P(g)$ is the set of periodic points of $g$. It is proved by Hayashi [8] that $f \in \mathcal{F}(M)$ if and only if $f$ satisfies both Axiom A and the no-cycle condition.

Let $\Lambda$ be a closed $f \in \operatorname{Diff}(M)$-invariant set. We say that $\Lambda$ is hyperbolic if the tangent bundle $T_{\Lambda} M$ has a $D f$-invariant splitting $E^{s} \oplus E^{u}$ and there exist constants $C>0$ and $0<\lambda<1$ such that

$$
\left\|\left.D_{x} f^{n}\right|_{E_{x}^{s}}\right\| \leq C \lambda^{n} \quad \text { and } \quad\left\|\left.D_{x} f^{-n}\right|_{E_{x}^{u}}\right\| \leq C \lambda^{n}
$$

for all $x \in \Lambda$ and $n \geq 0$. If $\Lambda=M$, then we say that $f$ is an Anosov diffeomorphism.

\section{Statement of the results}

A fundamental problem in differentiable dynamical systems is to understand how a robust dynamic property on the underlying manifold would influence the behavior of the tangent map on the tangent bundle. For instance, in [9], Mañé proved that any $C^{1}$ structurally 
stable diffeomorphism is an Axiom A diffeomorphism. And in [10], Palis extended this result to $\Omega$-stable diffeomorphisms.

Let $M$ be a compact $C^{\infty} n$-dimensional Riemannian manifold endowed with a volume form $\omega$. Let $\mu$ denote the measure associated with $\omega$ that we call the Lebesgue measure, and let $d$ denote the metric induced by the Riemannian structure. Denote by $\operatorname{Diff}_{\mu}(M)$ the set of diffeomorphisms which preserves the Lebesgue measure $\mu$ endowed with the $C^{1}$-topology. In the volume-preserving case, the Axiom A condition is equivalent to the diffeomorphism being Anosov, since $\Omega(f)=M$ by the Poincaré recurrence theorem.

We define the set $\mathcal{F}_{\mu}(M)$ as the set of diffeomorphisms $f \in \operatorname{Diff}_{\mu}(M)$ which has a $C^{1}$ neighborhood $\mathcal{U}(f) \subset \operatorname{Diff}_{\mu}(M)$ such that for any $g \in \mathcal{U}(f)$, every periodic point of $g$ is hyperbolic. Note that $\mathcal{F}_{\mu}(M) \subset \mathcal{F}(M)$ (see [11, Corollary 1.2]).

Very recently, Arbieto and Catalan [11] proved that if a volume-preserving diffeomorphism is contained in $\mathcal{F}_{\mu}(M)$, then it is Anosov. Indeed, at first they used the Mañé results ([9, Proposition II.1]). Then they showed that $\overline{P(f)}$ is hyperbolic, where $P(f)$ is the set of periodic points of $f$. And they proved that the nonwandering set $\Omega(f)=\overline{P(f)}$ by Pugh's closing lemma. Finally, by the Poincaré recurrence theorem, $\Omega(f)=M$. From the above facts, we can restate the theorem as follows.

Theorem 2.1 Any diffeomorphism in $\mathcal{F}_{\mu}(M)$ is Anosov.

In [12], Lee showed that if a volume-preserving diffeomorphism belongs to the $C^{1}$ interior expansive or $C^{1}$-interior shadowing property, then it is Anosov. And [13] proved that if a volume-preserving diffeomorphism belongs to the $C^{1}$-interior weak shadowing property or $C^{1}$-interior weak limit shadowing property, then it is Anosov. From these results, we study the cases when a volume-preserving diffeomorphism $f$ is in $C^{1}$-interior various inverse shadowing property with respect to the class $\mathcal{T}_{d}(f)$, then it is Anosov. Let $\operatorname{int} \mathcal{I S}_{\mu}(M)$ denote the set of volume-preserving diffeomorphisms in $\operatorname{Diff}_{\mu}(M)$ satisfying the inverse shadowing property with respect to the class $\mathcal{T}_{d}$, and let int $\mathcal{W} \mathcal{I} \mathcal{S}_{\mu}(M)$ (respect., int $\mathcal{O I} \mathcal{S}_{\mu}(M)$ ) denote the set of volume-preserving diffeomorphisms in $\operatorname{Diff}_{\mu}(M)$ satisfying the weak inverse shadowing property with respect to the class $\mathcal{T}_{d}$ (respect., the orbital inverse shadowing property with respect to the class $\mathcal{T}_{d}$ ). From now, we only consider the class $\mathcal{T}_{d}$ when we mention the inverse shadowing property; that is, the inverse shadowing property' implies the 'inverse shadowing property with respect to the class $\mathcal{T}_{d}$. Now we are in a position to state the theorem of our paper.

Theorem 2.2 Let $f \in \operatorname{Diff}_{\mu}(M)$. We have

$$
\operatorname{int} \mathcal{I} \mathcal{S}_{\mu}(M)=\operatorname{int} \mathcal{O} \mathcal{I S}_{\mu}(M)=\operatorname{int} \mathcal{W} \mathcal{I} \mathcal{S}_{\mu}(M)=\mathcal{A} \mathcal{N}_{\mu}(M)
$$

where $\mathcal{A N}_{\mu}(M)$ is the set of Anosov volume-preserving diffeomorphisms in $\operatorname{Diff}_{\mu}(M)$.

\section{Proof of Theorem 2.2}

Let $M$ be a compact $C^{\infty} n$-dimensional Riemannian manifold endowed with a volume form $\omega$, and let $f \in \operatorname{Diff}_{\mu}(M)$. To prove the results, we will give the following well-known Franks lemma for the conservative case, stated and proved in [14, Proposition 7.4]. 
Lemma 3.1 Let $f \in \operatorname{Diff}_{\mu}^{1}(M)$ and $\mathcal{U}$ be a $C^{1}$-neighborhood of $f$ in $\operatorname{Diff}_{\mu}^{1}(M)$. Then there exist a $C^{1}$-neighborhood $\mathcal{U}_{0} \subset \mathcal{U}$ off and $\epsilon>0$ such that if $g \in \mathcal{U}_{0}$, for any finite $f$-invariant set $E=\left\{x_{1}, \ldots, x_{m}\right\}$, any neighborhood $U$ of $E$ and any volume-preserving linear maps $L_{j}: T_{x_{j}} M \rightarrow T_{g\left(x_{j}\right)} M$ with $\left\|L_{j}-D_{x_{j}} g\right\| \leq \epsilon$ for all $j=1, \ldots, m$, there is a conservative diffeomorphism $g_{1} \in \mathcal{U}$ coinciding with $f$ on $E$ and out of $U$, and $D_{x_{j}} g_{1}=L_{j}$ for all $j=1, \ldots, m$.

Remark 3.2 From the Moser theorem (see [15]), there is a smooth conservative change of coordinates $\varphi_{x}: U(x) \rightarrow T_{x} M$ such that $\varphi_{x}(x)=\overrightarrow{0}$, where $U(x)$ is a small neighborhood of $x \in M$.

Proposition 3.3 Iff $\in \operatorname{int} \mathcal{I S}_{\mu}(M)$, then every periodic point of $f$ is hyperbolic.

Proof Take $f \in \operatorname{int} \mathcal{I S}_{\mu}(M)$ and $\mathcal{U}(f)$ is a $C^{1}$-neighborhood of $f \in \operatorname{int} \mathcal{I S}_{\mu}(M)$. Let $\epsilon>0$ and $\mathcal{V}(f) \subset \mathcal{U}_{0}(f)$ be a corresponding number and a $C^{1}$-neighborhood given by Lemma 3.1. Suppose that there exists a nonhyperbolic periodic point $p \in P(g)$ for some $g \in \mathcal{V}(f)$. To simplify the notation of the proof, we may assume that $g(p)=p$. Then there is at least one eigenvalue $\lambda$ of $D_{p} g$ such that $|\lambda|=1$. Denote by $E_{p}^{c}$ the eigenspace corresponding to $\lambda$. Then we see that if $\lambda \in \mathbb{R}$, then $\operatorname{dim} E_{p}^{c}=1$, and if $\lambda \in \mathbb{C}$, then $\operatorname{dim} E_{p}^{c}=2$.

First, we consider $\operatorname{dim} E_{p}^{c}=1$. For simplicity, we may assume that $\lambda=1$ (the other case is similar). By making use of Lemma 3.1, we linearize $g$ at $p$ with respect to the Moser theorem; that is, by choosing $\alpha>0$ sufficiently small, we construct $g_{1} C^{1}$-nearby $g$ such that

$$
g_{1}(x)= \begin{cases}\varphi_{p}^{-1} \circ D_{p} g \circ \varphi_{p}(x) & \text { if } x \in B_{\alpha}(p), \\ g(x) & \text { if } x \notin B_{4 \alpha}(p) .\end{cases}
$$

Then $g_{1}(p)=g(p)=p$. Since the eigenvalue $\lambda$ of $D_{p} g_{1}$ is one, $D_{p} g_{1}(v)=v$ for any $v \in E_{p}^{c}(\alpha)$. Take $v_{0} \in E_{p}^{c}(\alpha)$ such that $\left\|v_{0}\right\|=\alpha / 4$. Then we set

$$
\mathcal{I}_{v_{0}}=\left\{t \cdot v_{0}:-1 \leq t \leq 1\right\} \subset \varphi_{p}\left(B_{\alpha}(p)\right) .
$$

Take $\epsilon_{1}=\alpha / 8$. Let $0<\delta<\epsilon_{1}$ be the number of the inverse shadowing property of $g_{1}$. Then by our construction of $g_{1}, \varphi_{p}^{-1}\left(\mathcal{I}_{\nu_{0}}\right) \subset B_{\alpha}(p)$. Put $\mathcal{J}_{p}=\varphi_{p}^{-1}\left(\mathcal{I}_{\nu_{0}}\right)$. Then we see that $g_{1}\left(\mathcal{J}_{p}\right)=\mathcal{J}_{p}$ and it is the identity map. For the above $\delta>0$, we can define $\mathcal{T}_{d}\left(g_{1}\right)$-method as follows. For any $x \in M$, we set $\varphi_{p}(x)=\left(v_{1}, \ldots, v_{n}\right)$ and $D_{p} g\left(v_{1}, \ldots, v_{n}\right)=\left(v_{1}, A\left(v_{2}, \ldots, v_{n}\right)\right)$. Here $A$ is corresponding to $|\lambda| \neq 1$. Then we define

$$
h(x)=\left(\varphi_{p}^{-1}\left(v_{1}\right)+\delta / 2, \varphi_{p}^{-1}\left(A\left(v_{2}, \ldots, v_{n}\right)\right)\right)=\left(x_{1}+\delta / 2, x^{\prime}\right)
$$

where $\varphi_{p}^{-1}\left(A\left(v_{2}, \ldots, v_{n}\right)\right)=x^{\prime}=\left(x_{2}, \ldots, x_{n}\right)$. Clearly, $d_{1}\left(g_{1}, h\right)<\delta$, and $h \in \mathcal{T}_{d}\left(g_{1}\right)$. Let $p$ be identified with $\mathbf{0}=(0, \ldots, 0)$. Then choose $x=\left(x_{1}, 0, \ldots, 0\right) \in \mathcal{J}_{p}$ such that $d(\mathbf{0}, x)=2 \epsilon_{1}$. Then

$$
d\left(h^{i}(\mathbf{0}), g_{1}^{i}(x)\right)=d(\mathbf{0}, x)=2 \epsilon_{1} .
$$

Thus, $g_{1}$ does not have the inverse shadowing property. 
We take a point $y=\left(y_{1}, 0, \ldots, 0\right) \in \mathcal{J}_{p}$ such that $d(y, x)<\epsilon_{1}$ and $h^{i}(y)=\left(\varphi_{p}^{-1}\left(w_{1}\right)+\right.$ $\left.i \delta / 2, \varphi_{p}^{-1}\left(A^{i}\left(w_{2}, \ldots, w_{n}\right)\right)\right)=\left(y_{1}+i \delta / 2, y^{\prime i}\right)$, where $\varphi_{p}(y)=\left(w_{1}, \ldots, w_{n}\right), y^{\prime}=\varphi_{p}^{-1}\left(A\left(w_{2}, \ldots, w_{n}\right)\right)$ and $A$ corresponding to $|\lambda| \neq 1$. Then $d\left(g^{i}(x), h^{i}(y)\right)=d\left(x, y_{1}+i \delta / 2\right)$ and for some $k \in \mathbb{Z}$,

$$
d\left(x, y_{1}+k \delta / 2\right)>\alpha / 8=\epsilon_{1} .
$$

This is a contradiction.

Therefore, we can choose a point $y \in M \backslash \mathcal{J}_{p}$ such that $d(x, y)<\epsilon_{1}$. Since $d\left(g_{1}^{i}(x), h^{i}(y)\right)=$ $d\left(\left(x_{1}, 0, \ldots, 0\right),\left(y_{1}+i \delta / 2, y^{\prime i}\right)\right)$, we can find $k \in \mathbb{Z}$ such that

$$
d\left(\left(x_{1}, 0, \ldots, 0\right),\left(y_{1}+k \delta / 2, y^{\prime k}\right)\right)>\epsilon_{1},
$$

where $y^{\prime k}=\varphi_{p}^{-1}\left(A^{k}\left(w_{1}, \ldots, w_{n}\right)\right)$. This is a contradiction since $f \in \operatorname{int} \mathcal{I S}_{\mu}(M)$.

Finally, if $\lambda \in \mathbb{C}$, then $\operatorname{dim} E_{p}^{c}=2$. To avoid the notational complexity, we may assume that $g(p)=p$. As in the first case, by Lemma 3.1, there are $\alpha>0$ and $g_{1} \in \mathcal{V}(f)$ such that $g_{1}(p)=g(p)=p$ and

$$
g_{1}(x)= \begin{cases}\varphi_{p}^{-1} \circ D_{p} g \circ \varphi_{p}(x) & \text { if } x \in B_{\alpha}(p), \\ g(x) & \text { if } x \notin B_{4 \alpha}(p) .\end{cases}
$$

With a $C^{1}$-small modification of the map $D_{p} g$, we may suppose that there is $l>0$ (the minimum number) such that $D_{p} g^{l}(v)=v$ for any $v \in \varphi_{p}\left(B_{\alpha}(p)\right) \subset T_{p} M$. Take $v_{0} \in \varphi_{p}\left(B_{\alpha}(p)\right)$ such that $\left\|v_{0}\right\|=\alpha / 4$, and set

$$
\mathcal{L}_{p}=\varphi_{p}^{-1}\left(\left\{t \cdot v_{0}: 1 \leq t \leq 1+\alpha / 4\right\}\right) .
$$

Then $\mathcal{L}_{p}$ is an arc such that

- $g_{1}^{i}\left(\mathcal{L}_{p}\right) \cap g_{1}^{j}\left(\mathcal{L}_{p}\right)=\emptyset$ for $0 \leq i \neq j \leq l-1$,

- $g_{1}^{l}\left(\mathcal{L}_{p}\right)=\mathcal{L}_{p}$, and

- $\left.g_{1}^{l}\right|_{\mathcal{L}_{p}}$ is the identity map.

Note that $g_{1}$ has the inverse shadowing property if and only if $g_{1}^{k}$ has the inverse shadowing property for all $k \in \mathbb{Z}$ (see [6]). As in the first case, we can show that $g_{1}$ does not have the inverse shadowing property, which contradicts the fact that $g_{1} \in \mathcal{U}(f)$. Thus, every periodic point of $f \in \operatorname{int} \mathcal{I S}_{\mu}(M)$ is hyperbolic.

Proposition 3.4 If $\in \operatorname{int} \mathcal{W I S} \mathcal{S}_{\mu}(M)$, then every periodic point off is hyperbolic.

Proof Take $f \in \operatorname{int} \mathcal{W} \mathcal{I} \mathcal{S}_{\mu}(M)$, and $\mathcal{U}(f)$ is a $C^{1}$-neighborhood of $f \in \operatorname{int} \mathcal{W} \mathcal{I} \mathcal{S}_{\mu}(M)$. Let $\epsilon>0$ and $\mathcal{V}(f) \subset \mathcal{U}_{0}(f)$ be a corresponding number and a $C^{1}$-neighborhood given by Lemma 3.1. Suppose that there exists a nonhyperbolic periodic point $p \in P(g)$ for some $g \in \mathcal{V}(f)$. To simplify the notation of the proof, we may assume that $g(p)=p$. Then, as in the proof of Proposition 3.3, we can take $\alpha>0$ sufficiently small and a smooth map $\varphi_{p}: B_{\alpha}(p) \rightarrow T_{p} M$. Then we can make an arc $\mathcal{J}_{p} \subset B_{\alpha}(p)$ and for some $g_{1} \in \mathcal{V}(f)$. Take $\epsilon_{1}=\left(\right.$ length $\left.\mathcal{J}_{p}\right) / 4$. Let $0<\delta<\epsilon_{1}$ be the number of the weak inverse shadowing property for some $g_{1}$. Then we can construct a map $h \in \operatorname{Diff}(M)$ as in the proof of Proposition 3.3. 
Let $p$ be identified with $\mathbf{0}=(0, \ldots, 0)$. Then choose a point $x=\left(x_{1}, 0, \ldots, 0\right) \in \mathcal{J}_{p}$ such that $d(\mathbf{0}, x)=2 \epsilon_{1}$. Since $g_{1}$ has the weak inverse shadowing property,

$$
\mathcal{O}_{h}(\mathbf{0}) \subset B_{\epsilon_{1}}\left(\mathcal{O}_{g_{1}}(x)\right)
$$

However, for any $y \in \mathcal{J}_{p}$,

$$
g_{1}^{i}(y)=y \quad \text { and } \quad h^{i}(y)=\left(y_{1}+i \delta / 2, y^{\prime i}\right)
$$

where $y^{\prime i}=\varphi_{p}^{-1}\left(A^{i}\left(w_{2}, \ldots, w_{n}\right)\right)$ is as in the proof of Proposition 3.3. Thus, it is easily seen that

$$
\begin{aligned}
& \mathcal{O}_{h}(\mathbf{0}) \not \subset B_{\epsilon_{1}}\left(\mathcal{O}_{g_{1}}(x)\right) . \\
& \text { If } y=\left(y_{1}, 0, \ldots, 0\right) \in \mathcal{J}_{p} \backslash\{p\}, \text { then } \\
& h^{k}(y)=\left(y_{1}+k \delta / 2, y^{\prime k}\right)=\left(y_{1}+k \delta / 2,0\right),
\end{aligned}
$$

where $y^{\prime k}=\varphi_{p}^{-1}\left(A^{k}\left(w_{2}, \ldots, w_{n}\right)\right)$. Thus, we know that

$$
\mathcal{O}_{h}(y) \not \subset B_{\epsilon_{1}}\left(\mathcal{O}_{g_{1}}(x)\right)
$$

This is a contradiction.

Finally, we can choose a point $y \in M \backslash \mathcal{J}_{p}$ such that $d(x, y)<\epsilon_{1}$. Then we know that

$$
h^{k}(y)=\left(y_{1}+k \delta / 2, y^{\prime k}\right)
$$

where $y^{\prime k}=\varphi_{p}^{-1}\left(A^{k}\left(w_{2}, \ldots, w_{n}\right)\right)$. Therefore,

$$
h^{l}(y) \notin B_{\epsilon_{1}}\left(\mathcal{J}_{p}\right),
$$

for some $l \in \mathbb{Z}$. Then

$$
\mathcal{O}_{h}(y) \not \subset B_{\epsilon_{1}}\left(\mathcal{O}_{g_{1}}(x)\right)=B_{\epsilon_{1}}(x)
$$

Thus, $g_{1}$ does not have the weak inverse shadowing property. This is a contradiction.

If $\lambda \in \mathbb{C}$, then as in the proof of Proposition 3.3, for $g_{1} \in \mathcal{V}(f)$, we can take $l>0$ such that $D_{p} g_{1}^{l}(v)=v$ for any $v \in \varphi_{p}\left(B_{\alpha}(p)\right) \subset T_{p} M$. As in the previous argument, we get a contradiction. Thus, every periodic point of $f \in \operatorname{int} \mathcal{W I S} \mathcal{S}_{\mu}(M)$ is hyperbolic. Consequently, if $f \in \operatorname{int} \mathcal{W} \mathcal{I S}_{\mu}(M)$, then $f \in \mathcal{F}_{\mu}(M)$.

Proposition 3.5 Iff $\in \operatorname{int} \mathcal{O I S}_{\mu}(M)$, then every periodic point off is hyperbolic.

Proof The proof is almost the same as that of Proposition 3.4. Indeed, let $f \in \operatorname{int} \mathcal{O} \mathcal{I S}_{\mu}(M)$ and $\mathcal{U}(f)$ be a $C^{1}$-neighborhood of $f \in \operatorname{int} \mathcal{O I S}_{\mu}(M)$. Let $\epsilon>0$ and $\mathcal{V}(f) \subset \mathcal{U}_{0}(f)$ be a corresponding number and a $C^{1}$-neighborhood given by Lemma 3.1. Suppose that there exists 
a nonhyperbolic periodic point $p \in P(g)$ for some $g \in \mathcal{V}(f)$. To simplify the notation of the proof, we may assume that $g(p)=p$. Then, as in the proof of Proposition 3.4, we can take $\alpha>0$ sufficiently small and a smooth map $\varphi_{p}: B_{\alpha}(p) \rightarrow T_{p} M$. Then we can make an arc $\mathcal{J}_{p} \subset B_{\alpha}(p)$ and for some $g_{1} \in \mathcal{V}(f)$. Take $\epsilon_{1}=\left(\right.$ length $\left.\mathcal{J}_{p}\right) / 4$. Let $0<\delta<\epsilon_{1}$ be the number of the orbital inverse shadowing property for some $g_{1}$. Then we can construct a map $h \in \operatorname{Diff}(M)$ as in the proof of Proposition 3.4. Let $p$ be identified with $\mathbf{0}=(0, \ldots, 0)$. Then choose a point $x=\left(x_{1}, 0, \ldots, 0\right) \in \mathcal{J}_{p}$ such that $d(\mathbf{0}, x)=2 \epsilon_{1}$. Since $g_{1}$ has the orbital inverse shadowing property,

$$
\mathcal{O}_{h}(\mathbf{0}) \subset B_{\epsilon_{1}}\left(\mathcal{O}_{g_{1}}(x)\right) \text { and } \quad \mathcal{O}_{g_{1}}(x) \subset B_{\epsilon_{1}}\left(\mathcal{O}_{h}(\mathbf{0})\right) \text {. }
$$

However, for any $y \in \mathcal{J}_{p}$,

$$
g_{1}^{i}(y)=y \quad \text { and } \quad h^{i}(y)=\left(y_{1}+i \delta / 2, y^{\prime i}\right)
$$

where $y^{\prime i}=\varphi_{p}^{-1}\left(A^{i}\left(w_{2}, \ldots, w_{n}\right)\right)=\left(y_{2}, \ldots, y_{n}\right)$ is as in the proof of Proposition 3.3. Thus, it is easily seen that

$$
\mathcal{O}_{h}(\mathbf{0}) \not \subset B_{\epsilon_{1}}\left(\mathcal{O}_{g_{1}}(x)\right)
$$

This is a contradiction since $f \in \operatorname{int} \mathcal{O I S}_{\mu}(M)$.

$$
\begin{aligned}
& \text { If } y=\left(y_{1}, 0, \ldots, 0\right) \in \mathcal{J}_{p} \backslash\{p\} \text {, then } \\
& \qquad h^{k}(y)=\left(y_{1}+k \delta / 2, y^{\prime k}\right)=\left(y_{1}+k \delta / 2,0\right),
\end{aligned}
$$

where $y^{\prime k}=\varphi_{p}^{-1}\left(A^{k}\left(w_{2}, \ldots, w_{n}\right)\right)$. Thus, we know that

$$
\mathcal{O}_{h}(y) \not \subset B_{\epsilon_{1}}\left(\mathcal{O}_{g_{1}}(x)\right)
$$

This is a contradiction.

Finally, we can choose a point $y \in M \backslash \mathcal{J}_{p}$ such that $d(x, y)<\epsilon_{1}$. Then we know that

$$
h^{k}(y)=\left(y_{1}+k \delta / 2, y^{\prime k}\right)
$$

where $y^{\prime k}=\varphi_{p}^{-1}\left(A^{k}\left(w_{2}, \ldots, w_{n}\right)\right)$. Therefore,

$$
h^{l}(y) \notin B_{\epsilon_{1}}\left(\mathcal{J}_{p}\right),
$$

for some $l \in \mathbb{Z}$. Then

$$
\mathcal{O}_{h}(y) \not \subset B_{\epsilon_{1}}\left(\mathcal{O}_{g_{1}}(x)\right)=B_{\epsilon_{1}}(x)
$$

Thus, $g_{1}$ does not have the orbital inverse shadowing property. This is a contradiction.

If $\lambda \in \mathbb{C}$, then as in the proof of Proposition 3.4, for $g_{1} \in \mathcal{V}(f)$, we can take $l>0$ such that $D_{p} g_{1}^{l}(v)=v$ for any $v \in \varphi_{p}\left(B_{\alpha}(p)\right) \subset T_{p} M$. As in the previous argument, in order to reach the same contradiction. Thus, every periodic point of $f \in \operatorname{int} \mathcal{O I S}_{\mu}(M)$ is hyperbolic. 


\section{Competing interests}

The author declares that they have no competing interests.

\section{Acknowledgements}

The author wishes to express their appreciation to the referee for their careful reading of the manuscript and valuable suggestions. This work is supported by Basic Science Research Program through the National Research Foundation of Korea (NRF) funded by the Ministry of Education, Science and Technology, Korea (No. 2011-0007649).

Received: 21 May 2012 Accepted: 13 November 2012 Published: 28 November 2012

\section{References}

1. Pilyugin, S: Shadowing in Dynamical Systems. Lecture Notes in Math., vol. 1706. Springer, Berlin (1999)

2. Corless, R, Pilyugin, S: Approximate and real trajectories for generic dynamical systems. J. Math. Anal. Appl. 189 409-423 (1995)

3. Choi, T, Lee, K, Zhang, Y: Chracterisations of $\Omega$-stability and structural stability via inverse shadowing. Bull. Aust. Math. Soc. 74, 185-196 (2006)

4. Diamond, P, Lee, K, Han, Y: Bishadowing and hyperbolicity. Int. J. Bifurc. Chaos Appl. Sci. Eng. 12, 1779-1788 (2002)

5. Kloeden, P, Ombach, J, Porkrovskii, A: Continuous and inverse shadowing. Funct. Differ. Equ. 6, 137-153 (1999)

6. Lee, K: Continuous inverse shadowing and hyperbolicity. Bull. Aust. Math. Soc. 67, 15-26 (2003)

7. Pilyugin, S: Inverse shadowing by continuous methods. Discrete Contin. Dyn. Syst. 8, 29-38 (2002)

8. Hayashi, S: Diffeomorphisms in $\mathcal{F}^{1}(M)$ satisfy Axiom A. Ergod. Theory Dyn. Syst. 12, 233-253 (1992)

9. Mañé, R: A proof of the $C^{1}$-stability conjecture. Publ. Math. IHÉS 66, 161-210 (1987)

10. Palis, J: On the $C^{1} \Omega$-stability conjecture. Publ. Math. IHÉS 66, 211-215 (1988)

11. Arbieto, A, Catalan, T: Hyperbolicity in the volume preserving scenario. Preprint

12. Lee, M: Volume preserving diffeomorphisms with expansive and shadowing. Preprint

13. Lee, M: Volume preserving diffeomorphisms with weak and weak limit shadowing. Preprint

14. Bonatti, C, Diáz, LJ, Pujals, ER: A C '-generic dichotomy for diffeomorphism: weak forms of hyperbolicity or infinitely many sinks or sources. Ann. Math. 116, 355-418 (2003)

15. Moser, J: On the volume elements on a manifold. Trans. Am. Math. Soc. 120, 286-294 (1965)

doi:10.1186/1029-242X-2012-275

Cite this article as: Lee: Volume-preserving diffeomorphisms with inverse shadowing. Journal of Inequalities and Applications 2012 2012:275.

\section{Submit your manuscript to a SpringerOpen ${ }^{\circ}$ journal and benefit from:}

- Convenient online submission

- Rigorous peer review

- Immediate publication on acceptance

Open access: articles freely available online

- High visibility within the field

- Retaining the copyright to your article 